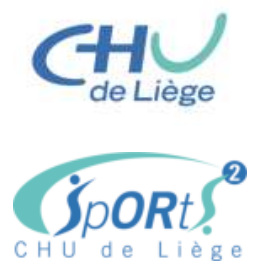

\title{
French cross-cultural adaptation and measurement properties of the "ANTERIOR KNEE PAIN SCALE"
}

F Buckinx¹, S Bornheim², G Remy2, J Van Beveren ${ }^{1}$, JY Reginster ${ }^{1}$,

O Bruyère ${ }^{1}$, N Dardenne ${ }^{1}$, JF Kaux ${ }^{2}$

1. Department of Public Health, Epidemiology and Health Economics, University of Liège, Liège, Belgium.

2. Physical Medicine and Sports Traumatology Department, University and University Hospital of Liège, Liège, Belgium.

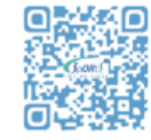

\section{Introduction}

Kujala Anterior Knee Pain Scale (AKPS), first developed in 1993, provides a functional assessment for knee complaints related to patellofemoral structure.

We aimed to linguistically and cross-culturally translate the AKPS into French and to evaluate the reliability and validity of this translated version of the questionnaire.

\section{Material and methods}

The translation part was performed in six stages, according to international guidelines: (i) two initial translations from English to French; (ii) synthesis of the two translations; (iii) backward translations into the original language; (iv) expert committee to compare the backward translations with the original questionnaire; (v) pre-final version testing and (VI) expert committee appraisal. To validate the French version of the Anterior Knee Pain Scale, we assessed its validity, reliability and floor/ ceiling effects. To do this, volunteer patients from the French part of Belgium and from France, with patellofemoral pain were asked to answer the French version of the Anterior Knee Pain Scale at baseline and after 7 days, as well as the generic SF-36 questionnaire.

\section{Results}

The Anterior Knee Pain Scale was translated without any major difficulties. A total of 101 subjects aged $34.5 \pm 11.4$ years (58.4\% of women) were included in this study. Results indicated an excellent test-retest reliability $(\mathrm{ICC}=0.97,95 \% \mathrm{Cl}$ 0.96-0.98), a high internal consistency (Cronbach's alpha $=0.87$ ), a consistent construct validity (high correlations with the SF-36 questionnaire were found with domains related to physical function $(r=0.80)$, physical role $(r=0.70)$, and pain $(r=0.64))$ and low or moderate correlations with domains related to mental health $(r=0.26)$, vitality $(r=0.32)$, and social function $(r=0.41))$. Moreover, no floor/ceiling effects have been found.

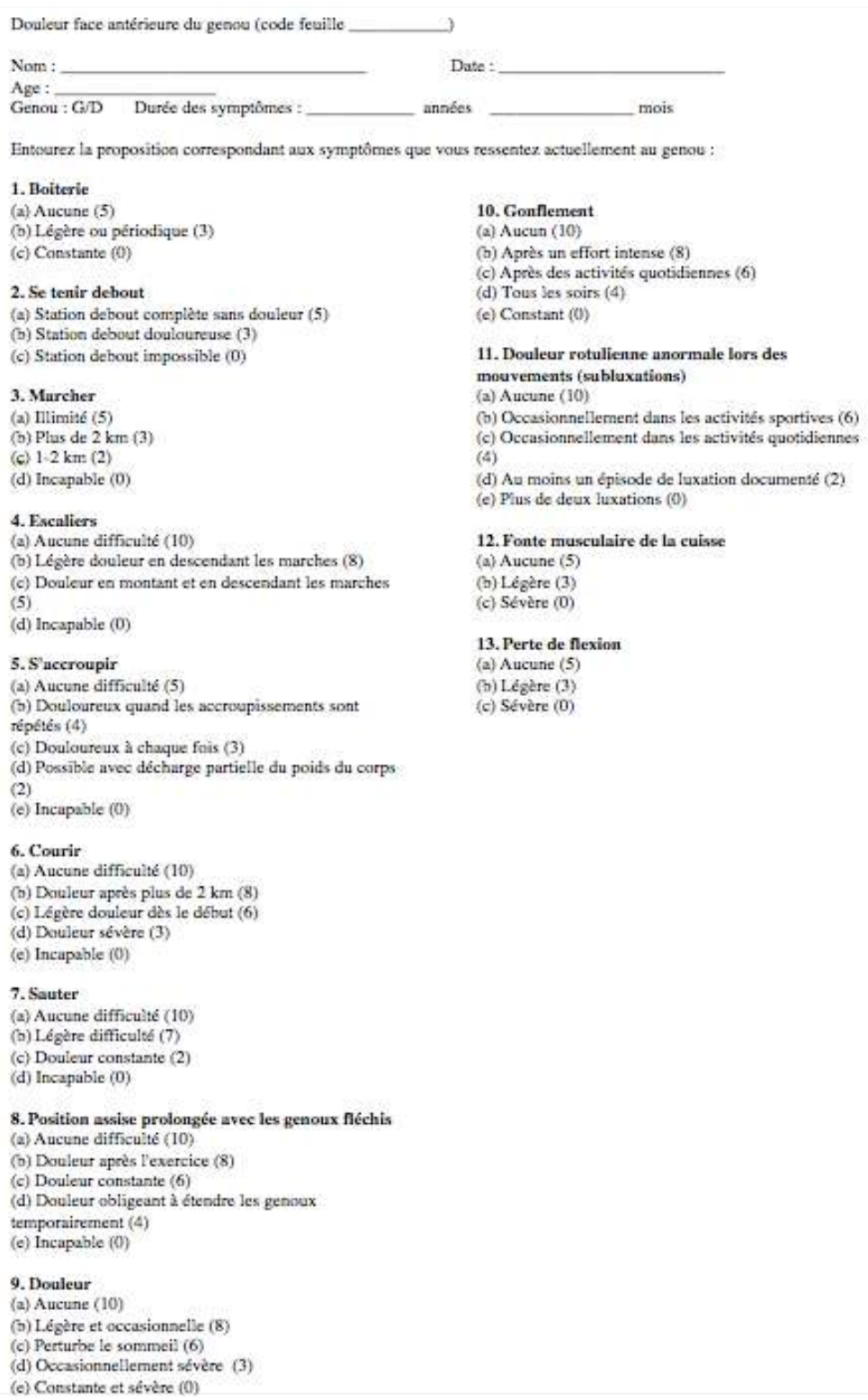

\section{Conclusion}

A valid French version of the Anterior Knee Pain Scale is now available and can be used with confidence to better assess the disease burden associated with patellofemoral pain. It was successfully cross-culturally adapted into French. 